\title{
Automatic detection and quantification of earthquake locations and basic source parameter for the Guyana M5,7 2021 earthquake sequence
}

Marcelo Bianchi', Caio Amaral', Jackson Calhau1, Daniel Rosa1, Javed Baksh², Daniella Assing², Christopher Basdeo², Emilia Brasilio', Bruno Collaço ${ }^{1}$, Marcelo Assumpção ${ }^{1}$

('Instituto de Astronomia, Geofísica e Ciências Atmosféricas/USP, Brazil; Guyana Geology and Mines Commission, Georgetown, Guyana)

Copyright 2021, SBGf - Sociedade Brasileira de Geofísica

This paper was prepared for presentation during the $17^{\text {th }}$ International Congress of the Brazilian Geophysical Society held in Rio de Janeiro, Brazil, 8-11 November 2021.

Contents of this paper were reviewed by the Technical Committee of the $17^{\text {th }}$ International Congress of the Brazilian Geophysical Society and do not necessarily represent any position of the SBGf, its officers or members. Electronic reproduction or storage of any part of this paper for commercial purposes without the written consent of the Brazilian Geophysical Society is prohibited.

\section{Abstract}

More than 3000 aftershocks (and foreshocks) were automatically located near the border of Brazil, part of a seismic sequence associated with the Guyana 2021 M5.7 earthquake. Data from BOAV station was processed using a template correlation while the local network stations were processed using a procedure based on STA/LTA detector with an AIC picker. Three catalogs were built containing origin times, magnitudes, epicenters and hypocenter when possible. Our best catalog readings presented a final standard error on $0.15 \mathrm{~s}$, estimated from a composed Wadatti diagram analysis. Near to the epicentral area we found a $\mathrm{Vp} / \mathrm{Vs}$ value equal to $1.671 \pm 0.006$. The $G-R$ relation analysis indicates that we obtained a completeness magnitude close to $1.4 \mathrm{MLV}$ with a "b-value" equals to $0.85 \pm 0.01$. From the hypocenter distribution we confirmed a NW-SE fault plane dipping to the SW direction.

\section{Introduction}

On 31 January 2021 a M5.7 earthquake struck the Deep South Guyana, $120 \mathrm{~km}$ east of the Boa Vista city of Roraima in Brazil with waves being well recorded by most of the Brazilian Seismographic Network (RSBR) and felt up to Manaus at $>600 \mathrm{~km}$ away. This earthquake was the largest earthquake so far recorded by the RSBR in the stable continental region of South America and was the main player of a sequence that initiated back in December 2020, and that continues to generate earthquakes in May 2021.

After the earthquake an initial group was set up by the Guyana Civil Defence (CDC) and the Guyana Geology and Mines Commission (GGMC) that was supported by the University of São Paulo Seismological Center to establish a local network (code XV) composed of 4 stations (GUY1, GUY2, GUY3 and GUY4) installed in the epicentral area, determined by the INSAR data analysis (Assumpção et al., 2021).

Using data from Boa Vista station (BOAV) and the local network stations we implemented an automatic procedure to obtain three different catalogs of events for later analysis. Catalogs described by this work are the result of a complete automatic procedure. We target to obtain, origin times, epicenters, hypocenters, magnitudes and, some initial source parameters, as the sequence b-value, additionally to the $\mathrm{Vp} / \mathrm{Vs}$ in the epicentral area.

\section{Method}

Continuous data from the BOAV station and the local network were used independently to derive three different catalogs. A regional and two local catalogs. The regional catalog contained only event time and magnitude (detections were not relocated). Local catalogs also determined the epicenter or hypocenter from each event. In comparison to the local offline Guyana network, BOAV is a broadband realtime station, with a low noise level that allowed us to detect the precursors and aftershocks, even before the installation of the local network. Guyana local network is composed of two shortperiod and two intermediate-period posthole stations. Data collection was made every week for the first month of operation. Today (May/2021) data is collected every month by GGMC personnel.

Table 1: Catalogs and catalog build parameters.

\begin{tabular}{|l|l|l|l|}
\hline & Regional & \multicolumn{1}{|c|}{ Local } & \multicolumn{1}{|c|}{ Local+ } \\
\hline Stations & BOAV & $\begin{array}{l}\text { Local (not } \\
\text { all) }\end{array}$ & Local (All) \\
\hline Method & $\begin{array}{l}\text { Template } \\
\left({ }^{*}\right)\end{array}$ & $\begin{array}{l}\text { STA/LTA } \\
\left({ }^{*}\right)\end{array}$ & $\begin{array}{l}\text { STA/LTA } \\
\left({ }^{* *}\right)\end{array}$ \\
\hline $\begin{array}{l}\text { Available } \\
\text { parameters }\end{array}$ & $\begin{array}{l}\text { Time \& } \\
\text { Magnitude }\end{array}$ & $\begin{array}{l}\text { Time, } \\
\text { Magnitude, } \\
\text { Epicenter }\end{array}$ & $\begin{array}{l}\text { Time, } \\
\text { Mygnitude, } \\
\text { Hypocenter }\end{array}$ \\
\hline Phases & S+Surface & $\begin{array}{l}\text { P and S on } \\
\text { any local }\end{array}$ & $\begin{array}{l}\text { P on all, S } \\
\text { on GUY3 } \\
\text { and GUY4 }\end{array}$ \\
\hline \# of Events & 2116 & 2490 & 851 \\
\hline
\end{tabular}

$\left({ }^{*}\right)$ Templates are filtered $(0.9-2.5 \mathrm{~Hz})$ waveforms of $15 \mathrm{~s}$ after the S-wave arrival for the event ids: usp2021cuaw, usp2021crio and usp2021cegw (Figure 1). Minimum detection correlation limit used was 0.55 for event usp2021cegw, and 0.35 for the others. An interval of $4 \mathrm{~s}$ was considered while unifying all template sub-catalogs. $\left({ }^{* *}\right)$ Short-/Long-term windows are 0.25 s and 20 s, Trigger on/off values were $4.5 / 1.2$ evaluated between $8-35 \mathrm{~Hz}$. Detections were all considered $\mathrm{P}$-wave arrivals that were grouped considering a $2.5 \mathrm{~s}$ search window between stations. AIC was applied on a window of $0.5 \mathrm{~s}$ around $\mathrm{P}$ and $2 \mathrm{~s}$ for the S-wave arriving no later than $3 \mathrm{~s}$ from P-wave and a minimum SNR of $1.5 .\left(^{* * *}\right)$ This catalog requires a $\mathrm{P}$-wave to be present on all local stations. 
Automatic detection of EARTHQuake in Guyana

BOAV continuous data was processed using a template correlation method (Chamberlain et al., 2017). We selected three different, $15 \mathrm{~s}$ waveforms templates (containing the S-wave, and Surfaces waveforms), generated from different events in the sequence (Figure 1). Template detections were not relocated, but assumed the location of the major shock, and had their magnitude determined using the SeisComp3 software package (Helmholtz-Centre Potsdam, 2008).
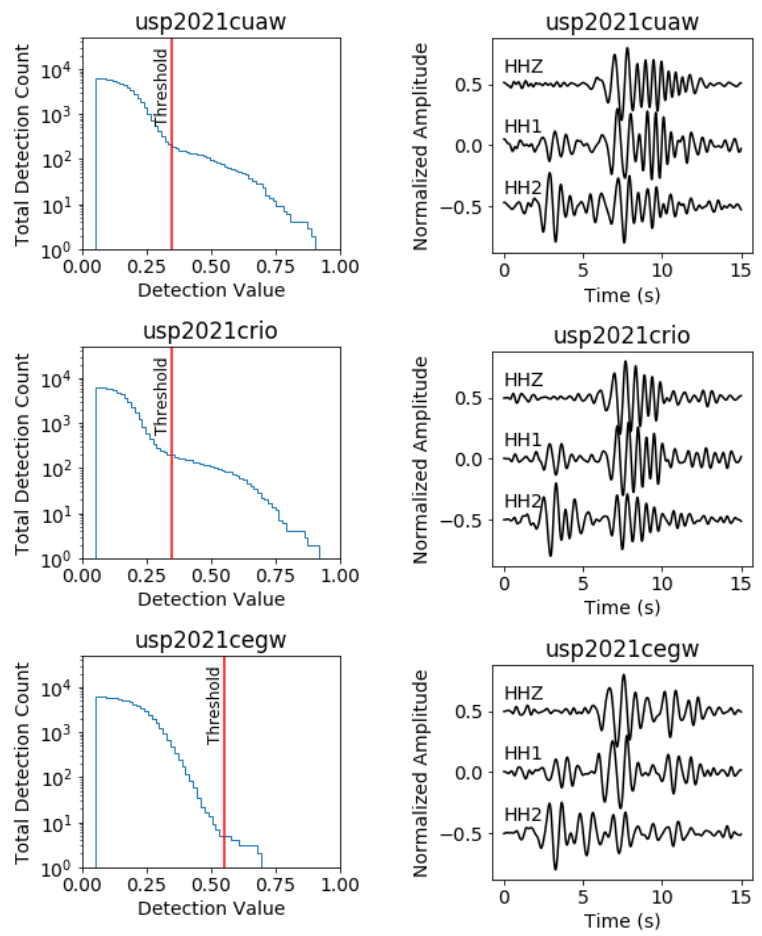

Figure 1: Detection limit analysis (left) and template waveforms for each event (right). Blue line represents the accumulated detection count by the threshold. Red line indicates chosen final thresholds. Lower thresholds allow the detection of noise signals, while higher values reduce the number of detections. Threshold limit tests used data from 31 March 2021. Event usp2021cuaw and usp2021cio magnitudes are 2.2 MLv and $4.3 \mathrm{mR}$ respectively. Event usp2021cegw is the main shock, with magnitude M5.7.

The local network was processed using a modified subset of SeisComp3 tools used in offline mode. We applied a STA/LTA detector, grouped the phase detections by time difference and later applied an AIC picker (St-Onge, 2011) for the P- and S-waves individually. Grouped picks were relocated using a local model with the standard Hypo71 locator to form the catalog. Any local event that could not be located was discarded and for the rest, the local MLv magnitudes were computed. Important parameters used for the template match, or the STA/LTA detection are documented by Table 1 . The velocity model used during the location consisted of a layer with $V p=5.88$ $\mathrm{km} / \mathrm{s}$ on top of a halfspace model of velocity $\mathrm{Vp}=6.4 \mathrm{~km} / \mathrm{s}$. A $V p / V s$ of 1.705 was considered, determined from a
Wadatti diagram using a set of 41 manual well located hypocenters (Assumpção et al., 2021).

As discussed above, templates are segments of 3-channels waveforms that were correlated to continuous data from BOAV station. For a template to declare a detection its correlation value has to be above a threshold that was determined by the inflection point of the accumulated number of detections by the detection limit curve (Figure 1, left). The test presented was performed for one day only (31 of January 2021) and was used to define the detection thresholds. Events cuaw and crio show a similar behaviour, with thresholds near $\sim 0.35$ while the main shock (cegw) has a slightly different waveform that matched only the larger aftershocks and needed a higher threshold than the others to operate. Detections below the inflection point show a higher probability of being false detections and were discarded.

\section{Results \& Discussions}

Each of the prepared templates (Figure 1) were correlated to all continuous data available from BOAV station from 01 of December 2020 to 31 of March 2021. Matched waveforms were used to determine the MLV magnitude for each new detected event. Template cuaw detected initially 5324 events, with magnitudes ranging from 0.7 MLv to 6.2 MLv. Template crio 4084 events with magnitudes between 0.8 MLv and 5.4 MLv and finally, template cegw detected only 215 events with magnitudes from 0.8 MLv to 6.1 MLv. Templates generated from lower magnitudes (2.2 MLv and 4.3 MLv) events were more efficient in detecting aftershocks while the main event template was not capable of detecting aftershocks, probably due to its greater magnitude and the increased content of low frequency due to its source spectra. Templates cuaw and cegw also detected false events, with magnitudes up to $6.1 \mathrm{MLv}$ that were later discarded from the processing.

To analyse the catalog completeness we show in Figure 2 plots of the log of the number of events by magnitude (G-R relation) as detected from each of the templates. G-R parameters "a" and "b" are indicated in each case. Figure 2 indicates that template cegw has a completeness magnitude $\sim 2 \mathrm{MLv}$ and a lower "a" value (small number of detections) while templates cuaw and crio show a complete catalog to almost 1.2-1.4 MLv. "b" values from all the templates analysed are below 1.0, being all three consistent between each other given the determined uncertainties. The $b$-value for the sequence is estimated as $0.85 \pm 0.01$. 
M. Bianchi, et al.

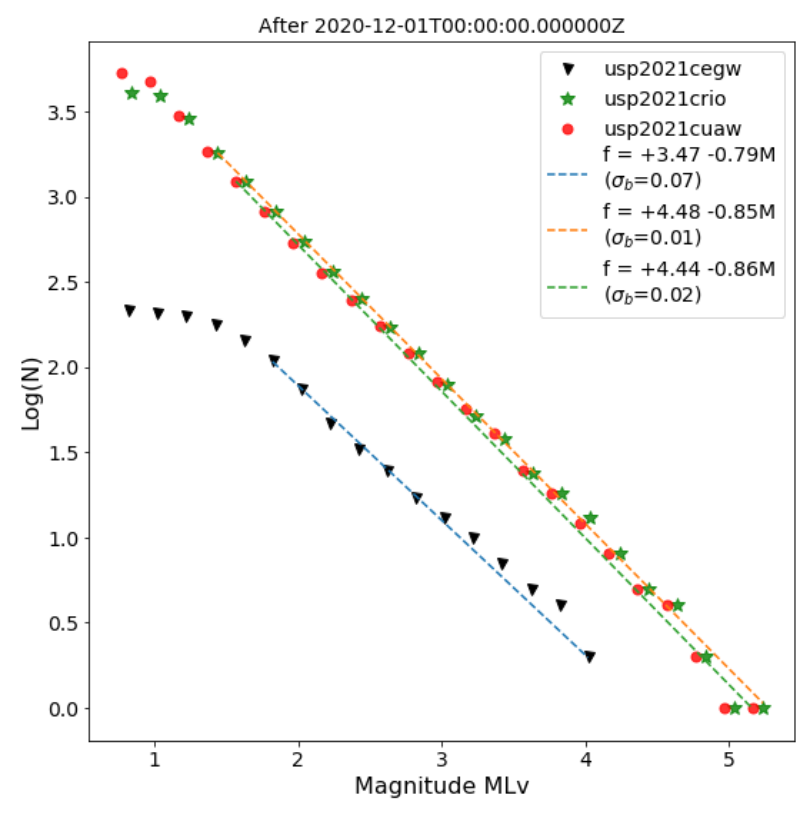

Figura 2: Gutenberg-Richter (G-R) relations fitted to the log of the number of events by Magnitude for all sets of events determined by the individual templates. Considered completeness was 1.4 for cuaw and crio and 1.8 for cegw. Fits parameters and uncertainties are indicated.

A final combined catalog was constructed from the individual set of detections from each template. The final template catalog contains 6204 events with magnitudes from 0.76 MLv to 5.36 MLv (as indicated, all events with magnitude larger than the main shock were discarded prior to the merge). When the magnitude range is restricted to a complete catalog confidence value, our final number of confirmed detections drops to 2116 . It is important to reinforce that BOAV station is more than 100 $\mathrm{km}$ from the epicentral area, and it would be very unlikely that it could detect such a large amount of low magnitude events.

The time evolution of both sequences, considering all detections (a) and only detections above the complete magnitude (b) as recorded by BOAV station is presented in Figure 3. To enhance the comparison we highlight the positions of all events with magnitude larger or equal to 3.5 MLv. Comparing sequences presented in Figure 3a and $3 \mathrm{~b}$ we conclude that: (i) the sequence started back in December 2020 with a set of events that lasted until around christmas time and that culminated to the 5.7 Guyana event of January 2021 (ii) while the density of small events (differences between Figure $3 a$ and $3 b$ ) follows the general trend presented in Figure $3 b$, it is hard to rule out the fact that a large part of those small events are false detections since there is a part of it that appears as a constant number of detections in time prior and after the mainshock. Nevertheless, the BOAV station analysis confirmed that the Guyana main event is part of a larger intraplate seismicity sequence that could be automatically detected and (partly) analysed with that only station. a)

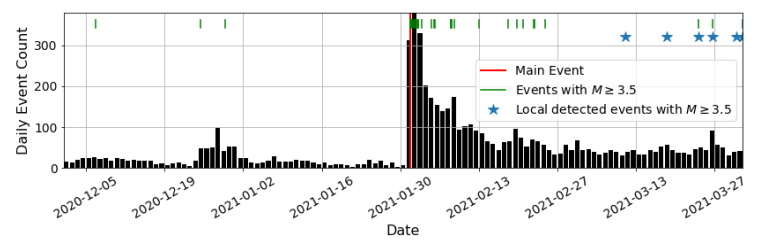

b)

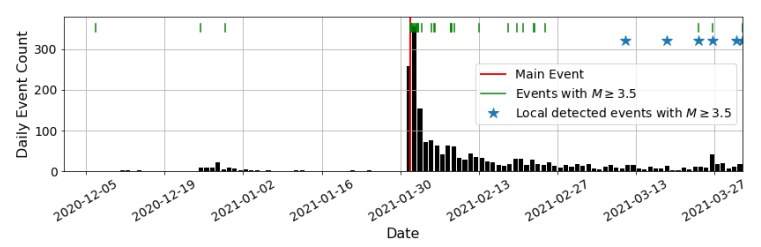

Figure 3: Temporal evolution of the number of events since 01 December 2020 for the total number of detections (a) and considering detection just above the completeness magnitude (b). A foreshock was observed in the middle to late December followed by the main sequence that started on 31 January 2021. Red and Green bars indicate the times of the main shock and foreshocks/aftershocks with magnitudes greater than 3.5 MLv. Green stars indicate the location of events larger than 3.5 MLv as detected by the local network. Each plot bar counts the total number of events in a day period.

With the installation of the local network (Figure 4) a different setup was available to detect events. We switched from the template correlation procedure to the classic STA/LTA method while making detections mainly due to the fact that to the network aperture, variations in the local positions of events were enough to generate completely different waveforms recorded. If we continue to use the correlation method, a much larger number of templates would be necessary.

Local network raw data processed with STA/LTA returned a total of 4 455, 7 293, 7005 and 22860 picks for stations GUY1, GUY2, GUY3 and GUY4 in the period of 05 of March to 04 of April 2021. Those picks were grouped into proposed origins using the $2.5 \mathrm{~s}$ time difference between stations, and divided into two catalogs: The Local, containing 3899 proposed origins detected in two or three stations simultaneously and the Local+, containing 863 proposed origins detected on all 4 stations. During this step all detections from STA/LTA are considered as $\mathrm{P}$-wave arrivals.

The next step consisted of refining the detections to generate picks using an AIC implemented procedure. We also implemented a search for a reasonable S-wave after the P-wave. Due to instrument noises on stations GUY1 and GUY2, and the fact that each proposed origin in Local+ catalog already had 4 picks, we opted to only search for S-wave picks on stations GUY3 and GUY4 for this catalog. Proposed origins on the Local catalog had the S-wave searched on all 4 stations while attempting to improve the number of proposed origins that could be promoted to origins. A proposed origin is promoted to an origin if it can be successfully relocated. Final located events are shown by the map and profile in Figure 4. 
Automatic detection of EARTHQUake In Guyana
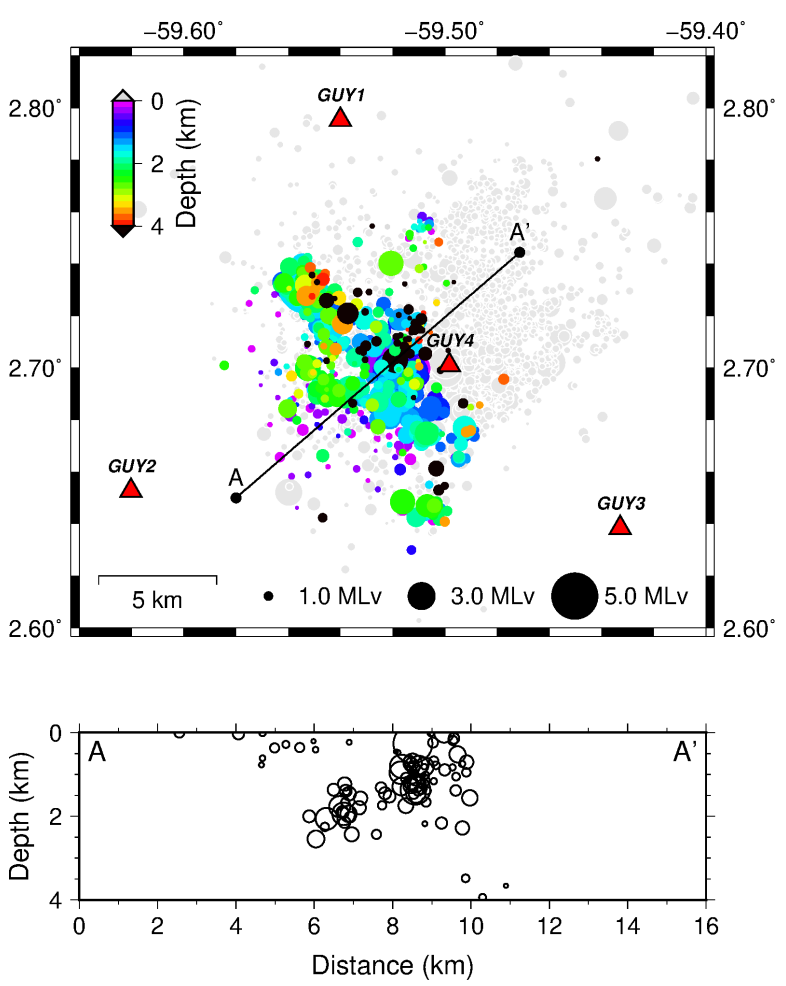

Figure 4: Catalog Local+ Hypocenter (depth colored) and Local Epicenter (gray). Profile A-A' considers the Local+ catalog up to $0.5 \mathrm{~km}$ away from the profile. Red Triangle are XV stations. Local+ hypocenters define a NW-SE fault dipping to the SW direction.

The map shown in Figure 4 shows all 851 and 2490 located origins from Local+ and Local catalogs. The Local catalog is plotted as gray circles that represents each epicenter position estimated by the location program. The depth was fixed to $2 \mathrm{~km}$ during the location. Local+ hypocenter are the depth color coded circles on the map, and the circles on the A-A' profile.

The Local catalog managed to migrate only $64 \%$ of the proposed origins to origins. This indicated that many of the considered picks in this catalog are badly grouped, or badly positioned in time. Further analyses and refinement will still be necessary to understand the reason for such a lower success rate.

The Local+ catalog migrated $99 \%$ of proposed origins to origins showing a well constrained result, mapping a dipping SW fault plane oriented in the NW-SE direction. On the other hand, the Local catalog while presenting many events within the bounds of the network was not able to constrain the fault plane with many bad events, incorrectly located to the North-East of the network, in the direction of the largest azimuthal-gap.

Finally, to analyse the quality of the Local+ catalog we computed a compound Wadati diagram adjusted for all the events that is shown by Figure 5. The final $\mathrm{Vp} / \mathrm{Vs}$, when all events are considered, was $1.671 \pm 0.006$ and the final standard error of all readings (excluding the outliers) was $0.154 \mathrm{~s}$.

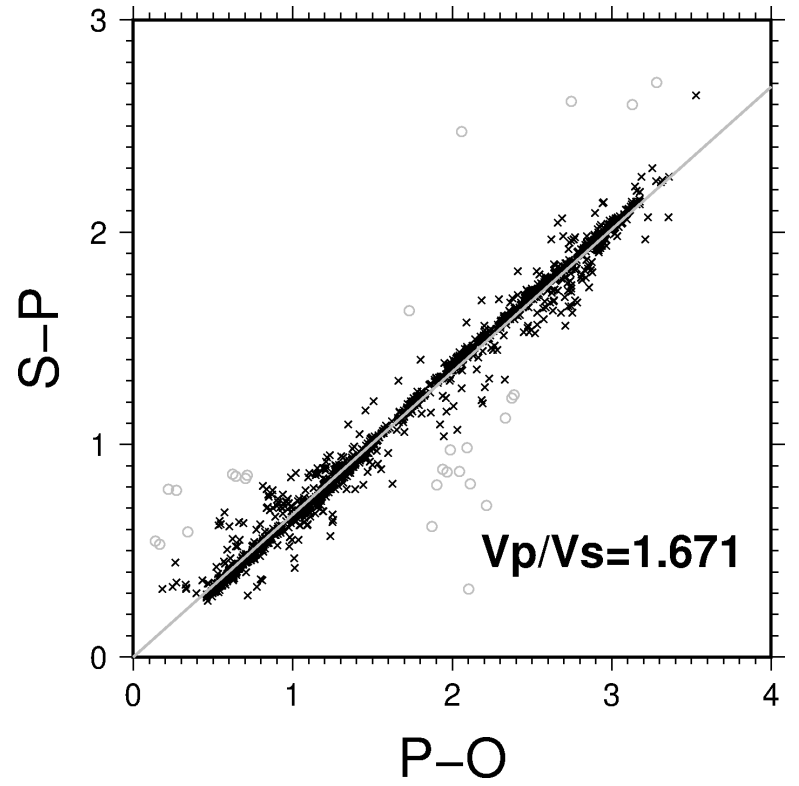

Figure 5: Composed Wadati diagram for the Local+ catalog. Gray circles are picks with errors larger than $2 \sigma$. The standard deviation of the readings dataset was estimated as $0.154 \mathrm{~s}$.

As a final remark, as can be observed by the map presented by the Flgure 4 the GUY4 station is the one closer to the fault and the one closer to the vast majority of the located events. It is also the station that presented the higher number of STA/LTA detections that could be indicating that we were able to locate just a fraction of the events that are occuring the Guyana epicentral area.

\section{Conclusions}

The efficient usage of standard techniques, implemented into open tools like SeisComp3 and EqCorrscan, allowed a detailed study of a local seismicity sequence that occurred in the stable South America with seismicity rates equivalent to any Andean seismicity sequence. Thousands of earthquakes were detected in a month, allowing for the fault plane directions to be determined, the $\mathrm{Vp} / \mathrm{Vs}$ for the region and also the "b" value for the sequence. All consistent to what is expected on a stable platform even if with high seismicity rates.

While studying detailed seismic sequences was not a planned RSBR capacity, BOAV station was of extreme importance in studying the sequence. The one station close to the epicentral area was sufficient to follow the sequence in time, while the local network permitted a detailed study of the fault plane and hypocentral distributions. Further studies should focus on stress drop and rupture dynamics if possible. Also a probable GT-5 or at least a pseudo GT-5 could be derived from this data that could be used to improve our knowledgement of the South American intraplate seismicity and even help to calibrate the RSBR further detections. 


\section{Acknowledgments}

M. Bianchi, et al.

The authors would like to thank all the support from the Guyana CDC involved in the deployment and operation of the local network.

\section{References}

Assumpção, M.; Calhau, J.; Rosa, D.; Bianchi, M.; Baksh, J.; Assing, D.; Basdeo, C.; October, S.; Persaud, L.; Jolivet, R.; Calais, E. The M5.7 earthquake of 31-January-2021, Guyana: preliminary field results of the largest recorded earthquake in the Guyana Shield, northern Amazon craton. The Seismic Record, Submitted, 2021.

Chamberlain, C. J., Hopp, C. J., Boese, C. M., Warren-Smith, E., Chambers, D., Chu, S. X., Michailos, K., Townend, J., EQcorrscan: Repeating and near-repeating earthquake detection and analysis in Python. Seismological Research Letters, 2017.

Helmholtz-Centre Potsdam - GFZ German Research Centre for Geosciences and GEMPA GmbH. The SeisComP seismological software package. GFZ Data Services, 2008. doi:10.5880/GFZ.2.4.2020.003.

St-Onge, A. (2011). Akaike information criterion applied to detecting first arrival times on microseismic data. In SEG Technical Program Expanded Abstracts. SEG Technical Program Expanded Abstracts 2011 (pp. 1658-1662). https://doi.org/doi:10.1190/1.3627522. 